\title{
Computed Tomographic Evaluation of Orbito-Ocular Injuries in Patients with Acute Head Trauma in University of Abuja Teaching Hospital Gwagwalada.
}

\author{
Kolade-Yunusa, Hadijat $\mathrm{O}^{1}$ \\ Department Of Radiology, University Of Abuja Teaching Hospital Gwagwalada, \\ Abuja Federal Capital Territory, Nigeria.
}

\begin{abstract}
:
Background: Associated orbito-ocular injuries do occasionally occur in acute head trauma patients. However there are scanty studies of patterns of orbito-ocular injuries in head trauma patient using computed tomography.

Aim :To determine the prevalence and pattern of orbital-ocular injuries in patients with acute head trauma. Methods :A retrospective review of one hundred and forty-three cases of acute head trauma sent for computed tomography evaluation over a period of twelve months (July 2015-July 2016) at the University of Abuja teaching hospital Gwagwalada, (UATH) Abuja FCT,Nigeria was carried out. The computed tomographic images and results were reviewed for presence of associated orbito-ocular injuries. Demographic characteristics of the patients, and detailed information about aetiologyand the severity of the head trauma were also recorded.

Results: A total of 143 patients comprising of 114(79.7\%) males and 29(20.3\%) females were included in the study with mean age of $31.76 \pm 17.45 y e a r s$. Majority of the patients $44(30.8 \%)$ were in the age group 21-30 years. $26.6 \%$ of patients with acute head trauma had ocular injuries while the remaining $73.4 \%$ were normal. Road traffic accidents (RTA) were the most predominant cause of head injury among the patients accounting for $77.6 \%$ of cases. $22.4 \%$ of patients with acute head trauma from RTA had ocular injuries accounting for the most common aetiology associated with ocular injuries. Ocular injuries were seen most in patients with moderate and severe closed head injury. Simple fracture was demonstrated in 13(9.1\%) patients and was the most common orbito-ocular findings.

Conclusion: The prevalence of orbito-ocular findings in acute head traumain this study was 26.6\%. This study has showed the usefulness of CT in evaluation of ocular injuries associated with head trauma. Computed tomography is highly recommended as the single most informative diagnostic modality for ocular injuries.
\end{abstract}

Keywords: computed tomography, ocular findings, acute head trauma

\section{Introduction}

Head trauma is any injury that causes any lesion or functional damage to the scalp, skull or brain. It is considered as a major health challenge causing death and disability, thus having considerable demands on the health services. In developing countries including Nigeria, accident rates in general and traumatic brain injury in particular are increasing because of increasing traffics load, poor states of the roads and the use of motor cycles as major means of transportation ${ }^{1,2}$. There are two general categories of head injuries:closed and penetrating. A closed injury is one in whichthe skull is not broken open. In penetrating injury, theskull is broken open.

Ocular injuries are usually associated with injuries to head and neck and injuries to the eyes can be directly or indirectly related to head trauma. Ocular injuries can present in isolation or as part ofpolytrauma. The associated displacement, stretching and shearing forces in head injury may damage areas of the brain including those associated with vision ${ }^{3}$. Also direct ocular trauma also contributes to the visual dysfunction in patients with head injury ${ }^{4}$.

Post-traumatic orbital injuries are classified into 2 groups as open and close globe injuries. However anterior chamber injuries, injuries to the lens, open-globe injuries, ocular detachments, intraorbital foreign bodies, orbital fractures, carotid cavernous fistula, and optic nerve injuries are common pathologies observed $^{4,5,6}$.

Definitive diagnosis of ocular trauma in the acute setting especially if patient is severely injured may be challenging clinically ${ }^{7}$ because most patients may be unable tocooperate or respond, making it difficult to evaluate the eyes for possible ocular injury,moreover fundoscopy may be impossible because eyes are swollen and shut. 
Missed ocular injuries in polytrauma patients may lead to serious morbidity later on, necessitating the need of high index of suspicion and employment of appropriate imaging techniques to accurately define the extent and type of ocular injury in other to prevent life time disabilities like visual impairment to complete loss of vision ${ }^{6}$. It has been estimated that world-wide, ocular injuries are responsible for blindness in approximately 1.6 million people ${ }^{8}$ and several retrospective studies have reported neuro-ophthalmic findings a few months after head trauma ${ }^{9,10}$. Early and prompt diagnosis of ocular injuries in head injury will go a long way in preventing these complications.Computed tomography scan and ultrasound are highly accurate in detection of ocular pathologies in ocular trauma ${ }^{11}$.

Computed tomography is considered as havingadvantage due to its ability of performing multiplanarreformation, evaluating intraorbital structures withsimultaneous imaging the bony orbit for fractures andany herniationof orbital contents ${ }^{12}$. Computed tomography is considered to be the top choice for evaluating orbital trauma. It offers short examination time and the best protocol is to obtain thin axial section ${ }^{6}$. Other imaging modalities include ultrasound for evaluating the globe and its content; however it is contraindicated in suspected globe ruptured. MRI has a better soft contrast with better visualization of the globe however it is contraindicated in patients with metallic intra-orbital foreign bodies ${ }^{6}$.This study is aimed at determining the prevalence and pattern of orbito-ocular findings in patient with acute head injury using computed tomography.

\section{Methodology}

Cranial CT scans of 143patients with varying degrees of acute head trauma obtained from July 2015July 2016 at University of Abuja teaching hospital Gwagwalada were reviewed retrospectively.Cranial CT examinations were done using the Toshiba Activion 16-slice CT scanners. A range of 120-140 kvp and 150-300 mAs were used for the cranial CT scans. Images were acquired in the axial plane at $2.5 \mathrm{~mm}$ from the base of the skull to the vertex with multiplanar reformatted sagittal and coronal images.Data reviewed from the request forms included age, sex, indication for the CT scan and degree of severity of the head injury. In addition patients whoseinformationwas not available were retrieved from the folder while those with incomplete information were discarded. A proforma was develop where the age, sex, age, severity of head trauma (mild, moderate, severe) and patterns of ocular findings on CT were recorded.

\section{Data analysis}

Statistical analysis was done using the Statistical Package for Social Sciences (SPSS) version 19.0. Simple frequencies were determined for the age and sex. The paired sample $t$-test was used for evaluating the significance, while the correlationwas determined using the Pearson correlation coefficient. A $P$-value below 0.05 was considered significant.

\section{Results}

A total of 143 subjects comprising $114(79.7 \%)$ males and 29(20.3\%) females were recruited for this study (Table 1). The mean age of the subjects was 31.76 \pm 17.45 years. The majority $44(30.8 \%)$ of the patients are in the age group of 21-30years (Table 1). The smallest proportion of $9(6.3 \%)$ of head trauma patients were seen in age group 51-60years. The mean age of males was 33.98 \pm 17.1 years and that of females was $23.03 \pm 16.3$ years. Acute head trauma was common among males compared to females in all age group (Table 1).

Acute moderate head injuryaccounted for majority $87(60.8 \%$ ) of all head trauma cases whileacute mild head injuryand acute severe head injuryaccounted for 21(14.7\%) and 35 (24.5\%) of head trauma respectively. Of the 87 subjects withacute moderate head injury 19(13.3\%) had ocular injury. 14(9.7\%) of acute severe head injury and $5(3.4 \%)$ subjects withacute mild head injuryhadocular injury respectively(Table 2).

RTA was the major cause of acute head injury accounting for 111(77.6\%) of cases.Cases of acute head trauma from falls, assault, occupational and gunshot accounted for 12(8.4\%), 14(9.8\%), 2(1.4\%) and 4(2.8\%) respectively. Occupational injury accounted for the least cause of head injury(Table 3). $22.4 \%$ of subjects with acute head trauma from RTA had ocular injuries accounting for the most common aetiology associated with ocular injuries(Table 3).38(26.6\%) of patients with acute head trauma had ocular injuries while the remaining 105 patients $(73.4 \%)$ were normal.There were no ocular injuries in majority of the patients. 31(2.6\%) of maleshad ocular injuries while 7 (4.9\%) females were noted with ocular injuries. Ocular injuries were found more in males than females(Table 4).

The patterns of ocular abnormalities were: complex fracture7 cases (4.9\%), simple fracture 13 cases (9.1\%), soft tissue swelling8 cases(5.6\%), ruptured globe 3 cases(2.1\%)and ocular emphysema 7 cases $(4.9 \%)$. Complex fracture refers to blow-out fractures and tripod fracture while simple fracture refers to simple linear fracture involving any of the walls of the orbit. Simple fracture was the most common orbito-ocular findings.Ocular emphysema was thesecond mostcommon finding(Table 4). 
Table 1: Age and sex distribution in patients with acute head trauma

\begin{tabular}{lccc}
\hline Age & Frequency (Percent) & Males (Percent) & Females (Percent) \\
\hline$<10$ & $14(9.8)$ & $5(3.5)$ & $9(6.3)$ \\
$11-20$ & $25(17.5)$ & $20(13.9)$ & $5(3.5)$ \\
$21-30$ & $44(30.8)$ & $37(25.9)$ & $7(4.9)$ \\
$31-40$ & $27(18.9)$ & $21(14.7)$ & $6(4.2)$ \\
$41-50$ & $13(9.1)$ & $13(9.1)$ & $0(0.0)$ \\
$51-60$ & $9(6.3)$ & $8(5.6)$ & $1(0.7)$ \\
$>60$ & $11(7.6)$ & $10(6.9)$ & $1(0.7)$ \\
& $143(100)$ & $114(79.7)$ & $29(20.3 \%)$
\end{tabular}

Table 2: Severity of head injury in relation to CT ocular findings

\begin{tabular}{lcccccccc}
\hline Severity & $\begin{array}{c}\text { Freq (\%) } \\
\text { Normal }\end{array}$ & $\begin{array}{c}\text { CT } \\
\text { Complex F }\end{array}$ & \multicolumn{2}{c}{ Findings } \\
& Simple F & STS RG & EMPHY & Total \\
\hline Mild & $21(14.7)$ & 16 & 0 & 3 & 2 & 0 & 0 & 21 \\
Moderate & $87(60.8)$ & 68 & 4 & 6 & 5 & 1 & 3 & 87 \\
Severe & $35(24.5)$ & 21 & 3 & 4 & 1 & 2 & 4 & 35 \\
Total & $143(100.0)$ & 105 & 7 & 13 & 8 & 3 & 7 & 143
\end{tabular}

Complex F=complex fracture, Simple F=simple fracture, STS=soft tissue swelling,

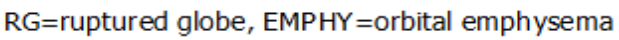

TABLE 3: CT ocular findings in relation to cause of head injury.

CAUSES OF HEAD INJURY

\begin{tabular}{|c|c|c|c|c|c|}
\hline CTFindings & RTA (\%) & Falls (\%) & Assault (\% & ) Occup ( & G.Sh (\%) \\
\hline Normal & 79 (55.2) & $11(7.7)$ & $11(7.7)$ & $1(0.7)$ & $3(2.1)$ \\
\hline Complex fracture & $6(4.2)$ & $0(0.0)$ & $1(0.7)$ & $0(0.0)$ & $0(0.0)$ \\
\hline Simple fracture & $10(7.0)$ & $1(0.7)$ & $1(0.7)$ & $0(0.0)$ & $0(0.0)$ \\
\hline Soft tissue swelling & $7(4.9)$ & $0(0.0)$ & $1(0.7)$ & $0(0.0)$ & $0(0.0)$ \\
\hline Ruptured globe & $3(2.1)$ & $0(0.0)$ & $0(0.0)$ & $0(0.0)$ & $0(0.0)$ \\
\hline \multirow[t]{2}{*}{ Orbital emphysema } & $6(4.2)$ & $0(00)$ & $0(0.0)$ & $0(0.0)$ & $1(0.7)$ \\
\hline & $111(77.6 \%)$ & $12(8.4 \%)$ & $14(9.8 \%)$ & $2(1.4 \%)$ & $4(2.8 \%)$ \\
\hline
\end{tabular}


Table4: CT ocular findings in relation to gender

\begin{tabular}{lccc}
\hline CT Findings & Freq. (Percent) & \multicolumn{2}{c}{ Sex } \\
& & Males (Percent) & Females (Percent) \\
\hline Normal & $105(73.4)$ & $83(72.8)$ & $22(75.9)$ \\
Complex fracture & $7(4.9)$ & $7(6.1)$ & $0(0.0)$ \\
Simple fracture & $13(9.1)$ & $12(10.5)$ & $1(3.4)$ \\
Soft tissue Swelling & $8(5.6))$ & $4(3.5)$ & $1(3.9)$ \\
Ruptured globe & $3(2.1)$ & $2(1.8)$ & $1(3.4)$ \\
Ocular emphysema & $7(4.9)$ & $6(5.3)$ & $29.3)$ \\
& $143(100.0)$ & $114(79.7)$ & \\
\hline
\end{tabular}

Computed tomographic imageof the orbit showing rupture of the left globe with associated fracture of the orbital wall. 


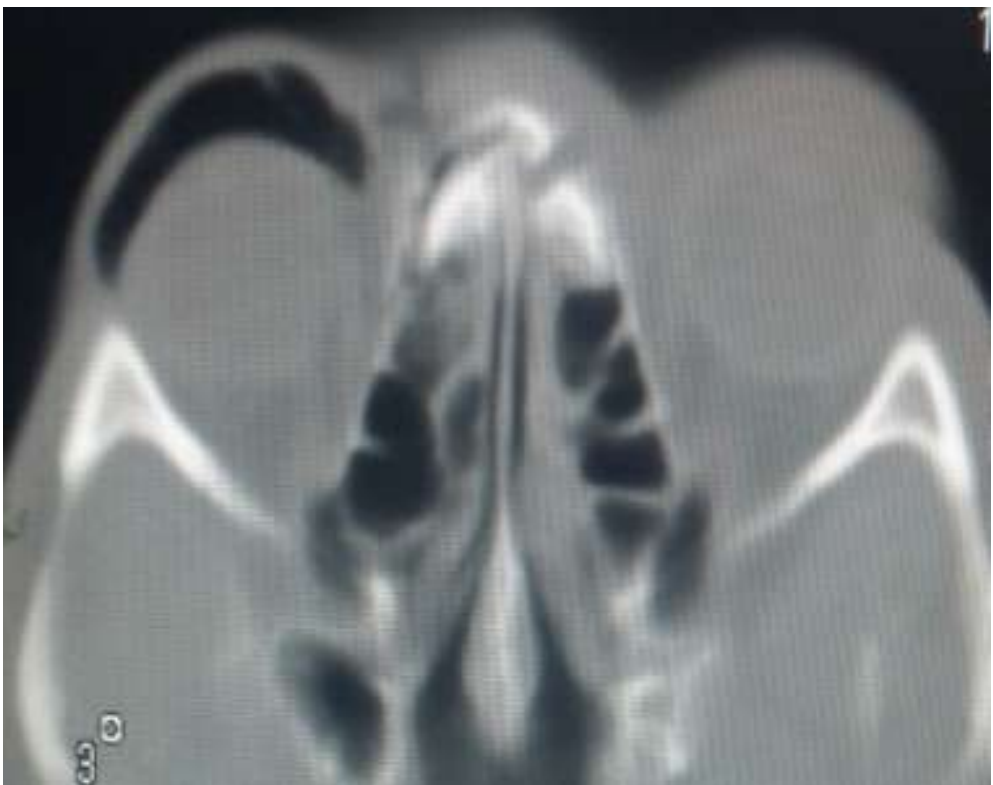

Figure 2

Computed tomographic imageof the orbit showing large right orbital emphysema with left soft tissue swelling.

\section{Discussion}

There has been scanty report on the use of computed tomography scans to detect associated orbitoocular injuriesin patients with acute head trauma. This prompted this study to determine the prevalence and patterns of associatedorbito-ocular injuries in acute head trauma. Computed tomography scan has been reported to be a very useful imaging modality in evaluating patients with suspected ocular injury which may have been missed during ophthalmology assessment of patients with acute head trauma. Prompt and early diagnosis of associated orbito-ocular injury in acute headtrauma is essential to prevent life complications like blindness.

Head trauma has been documented to be more prevalent in relatively young adults in general and male sex in particular.Expectedly the young adults were the most affected in this study, majority who are in their third decade of life werein age group 21-30years. The second highest age group was age group 31-40years followed by age group 11-20. Some other studies also corroborated this finding ${ }^{13-15}$. These can be explained by the fact that these age groups are the most active, who spend most of their time out of their houses for work to earn a livelihood and therefore are more prone to accidents. Majority of patient with head injury were males. Male to female ratio was 3.9:1. This was similar to what was obtained in studies by Salaam et $\mathrm{al}^{13}$ and Baker et al ${ }^{15}$.Male dominance is most probably due to the fact that males are more involved in outdoor activities and vehicular driving is still a male dominated activity in this part of the world.

Road traffic accident (RTA) was the major cause of head trauma among the patients accounting for $77.6 \%$ of the cases. It was therefore not surprising to see that a large proportion of patients $(22.4 \%)$ with head traumaassociated with ocular manifestations, RTA were responsible. This finding was also obtained in several other studies worldwide ${ }^{4,16-18}$. In this setting reasons for high rates of RTA are multifactorial however, bad roads, poorly maintained vehicles, lack of appropriate government policies on road safety rules, lack of adherence to traffic regulations by road users, speed limits are not strictly observed as well as unlicensed careless driving is treated with levity may all be responsible.

$73.4 \%$ of subjects did not have any abnormalocular findings in respective of the severity/degree of the head trauma accounting for the majority in this study. Similar findings were reported by Salaam et al ${ }^{13}$. This was contrary to what was obtained by Masilaet $\mathrm{al}^{4}$. The prevalence of ocular findings on computed tomography in patient with acute head injury in this study was $26.6 \%$. This was higher than value $(7.1 \%)$ obtained by Salaam et $\mathrm{al}^{13}$ in their work on Computed Tomography: Ocular manifestations in acute head injury patients in Jos University Teaching Hospital, but lower than value reported $\left(83.5 \%\right.$ )by Kulkarni et al ${ }^{17}$ in their study on ocularmanifestations of head injury. HoweverKulkarniet $\mathrm{al}^{17}$ included abnormal ocular findings noted clinically and on computed tomography in patients with head injury. The similarities of this present study with Salaamet $\mathrm{al}^{13}$ is ocular features in head trauma patients were limited to findings that were picked on Computed tomographyimaging.

Orbito-ocular injury was seen in patients irrespective of the degree of severity of the head trauma. However it was noticed that more severe injuries like complex fracture and ruptured globe occurred in patients with moderate and severe acute closed head injury patients while mild findings like soft tissue swelling and simple fractures were seen in mild closed head injury. This was contrary to findings by Salaam et al ${ }^{13}$. 
However, the similarity in both studies is that there was no significant correlation between presence of ocular injury and degree of head trauma.

Injuries to the globe and adnexa in the anterior segment of the eye in head trauma patient assuggested by a hypothesis may be probably due to the fact that energy is transferred to these structures from the sturdy frontal bones to the orbit and from the lateral orbital margin to contiguous facial structures during the impact following head injury ${ }^{19}$. Therefore globe ruptured may or may not be accompanied with orbital fracture. Fracture with concomitant globe ruptured was noted in one of the patients with severe acute head trauma.

Somestudieshave shown that anteriorsegment of the eye were more commonly involved in head injuries than the posterior segment ${ }^{18,20}$.Injuries to this segment result from direct impact on the rigid frontal bones and orbital margins. In this study simple fracture and soft tissue swelling accounted for majority oforbitoocular injuries in head trauma patient. Similar findings were obtained by Kanwal ${ }^{20}$ et al in their study on ocular manifestations associated with head injury. Occasionally simple fracture may be accompanied by soft tissue swelling and/or orbital emphysema. Most times these features were seen in isolation.

\section{Conclusion}

The prevalence of orbito-ocular injury in patients with acute head trauma using computed tomography in this study was $26.6 \%$. RTA was responsible as the etiological factor for these injuries. Majority of the ocular findings were seen in patients with moderate and severe head injury. Computed tomographic images of patient with head trauma should be well scrutinized for possible ocular injuries to prevent life time complications.

\section{References}

[1]. ChalyaLP,KanumbaSE,MabulaBJ,GiitiG,Gliyoma MJ. Aetiology, spectrum, injury charasteristics and treatment outcomeof head injury patients at Bugando medical center North-western Tanzania. Tanzania J. Health Res 2011.1:93-102 .

[2]. EmejuluJKC,EkweogwuCO,Nottidge T. The burden of motorcycle-related neurotrauma in South-east Nigeria. J.Clin.Med.Res.2009;1:13-17.

[3]. Taber KH, Warden DL, Hurley RA (2006). Blast related traumatic brain injury: what is known? J.Neuropsychiatr.Clin. Neurosci. 18: 141-5.

[4]. Masila F, Kiboi JG, Marco S, Njuguna M (2014). Ocular findings in patients with head injury. Int. J of Med. and Clin. Sciences, 1(2): 009-017.

[5]. Güzel M, Erenler AK, Niyaz L, Baydın A. Management of traumatic eye injuries in the emergency department. OA Emergency Medicine 2014 Jan 18;2(1):2.

[6]. Wayne S. Kubal, MD, Imaging of OrbitalTrauma. RadioGraphics 2008; 28:1729-1739.

[7]. Sadaf Imran, Saima Amin, M Imran HameedDaulaPakImaging in Ocular Trauma Optimizing the Use of Ultrasound and Computerised Tomography $J$ Ophthalmol2011; 3:146-151.

[8]. Negrel AD, Thylefors B. The global impact of eye injuries.OphthalmicEpidemiol. 1998; 5: 143-69.

[9]. Kowal L. Ophthalmic manifestations of head injury. Austra New Zealand J Ophthalmol 1992; 20: 35-40.

[10]. Van Stavern GP, Biousse V, Lynn MJ, Simon DJ, Newman NJ. Neuro-Ophthalmic manifestations of head trauma. J NeuroOphthalmol 2001;21(2): 112-117.

[11]. Blaivas M Theodor D, Sierzenski P. A study of bedside ocularultrasonography in the emergency department. AcadEmerMed. 2002; 9: 791-9.

[12]. DeramoVa, Shah Gk, Baumal CR, etal.Role of ultrasound biomicroscopy in ocular trauma. Trans Am Ophthamol Soc. 1998; 96: 355-65.

[13]. Salaam AJ, Aboje OA, Danjem SM, Ibinaiye PO, Tawe G S, Ekedigwe JE, Pam SD,Ani CC, Salaam AA. Computed Tomography: Ocular Manifestations In Acute Head Injury Patients In Jos University Teaching Hospital. Jos Journal of Medicine 2015,9:38-42 .

[14]. AdeyekunAA, Obi-Egbedi-EjakpoviEB. Computerised tomographic patterns in patients with head injury at the university of Benin teaching hospital. Niger JClinPrac 2013;16;19-22.

[15]. Baker RS Wilson MR, Flowers CW Jr, Lee DA, Wheeler NC.: Demographic factors in a population-based survey of hospitalized, work related, ocular injury. Am J Ophthalmol .1996;122:213-219.

[16]. Md. Ziya Ahmad, R. N. KarmakarAn Epidemiological Study of Acute Head Injury and It's Evaluation by CT scan. J Indian Acad Forensic Med. April-June 2014;36:173-175.

[17]. Kulkarni AR, Aggarwal SP. Ocular manifestations of head injury: A clinical study.Eye (London). 2005;19(12): 1257-63.

[18]. Odebode TO, Ademola-Popoola DS, Ojo TA, Ayanniyi AA. Ocular and visual complicationsof head injury. Eye. 2005 ; 19 : 561 566.

[19]. Duke Elder S, Wybar KC. The Anatomy of the Visual System. Eye and Sport Medicine. 1961; 2: 559-67 .

[20]. KanwalZareenAbbasi, BaseeratQadeer, Ali Raza.Ocular Manifestations Associated with Head Injury, Pak J Ophthalmol 2016;32:111-116 